LAWRENCE LIVERMORE NATIONAL LABORATORY

\title{
Shell Model in a First Principles Approach
}

P. Navratil

Janaury 15, 2004

The Sixth International Conference on Radioactive Nuclear Beams (RNB6) Argonne, IL

September 22-26, 2003 


\section{Disclaimer}

This document was prepared as an account of work sponsored by an agency of the United States Government. Neither the United States Government nor the University of California nor any of their employees, makes any warranty, express or implied, or assumes any legal liability or responsibility for the accuracy, completeness, or usefulness of any information, apparatus, product, or process disclosed, or represents that its use would not infringe privately owned rights. Reference herein to any specific commercial product, process, or service by trade name, trademark, manufacturer, or otherwise, does not necessarily constitute or imply its endorsement, recommendation, or favoring by the United States Government or the University of California. The views and opinions of authors expressed herein do not necessarily state or reflect those of the United States Government or the University of California, and shall not be used for advertising or product endorsement purposes. 
Shell model in a first principles approach

\author{
J. P. Vary ${ }^{\mathrm{a} *}$, B. R. Barrett ${ }^{\mathrm{b} \dagger}$, R. Lloyd $\mathrm{d}^{\mathrm{a} *}$, P. Navratic ${ }^{\ddagger \ddagger}$, A. Nogga $\mathrm{N}^{\mathrm{d}}$, W. E. Ormand ${ }^{\mathrm{c} \ddagger}$

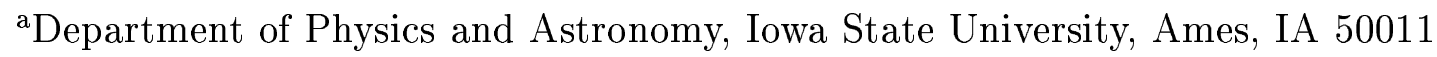 \\ ${ }^{\mathrm{b}}$ Department of Physics, University of Arizona, Tucson, AZ 85721 \\ ${ }^{\mathrm{c}}$ Lawrence Livermore National Laboratory, Livermore, CA 94551 \\ dinstitute for Nuclear Theory, University of Washington, Seattle, WA 98195
}

We develop and apply an ab-initio approach to nuclear structure. Starting with the NN interaction, that fits two-body scattering and bound state data, and adding a theoretical NNN potential, we evaluate nuclear properties in a no-core approach. For presently feasible no-core model spaces, we evaluate an effective Hamiltonian in a cluster approach which is guaranteed to provide exact answers for sufficiently large model spaces and/or sufficiently large clusters. A number of recent applications are surveyed including an initial application to exotic multiquark systems.

\title{
1. INTRODUCTION
}

Quantum many-body theory with a fixed number of Fermions has a rich history in Nuclear and Atomic Physics. In recent years, numerous applications in Particle Physics and Nano-physics have also arisen. Few approaches are capable of providing exact answers when the interactions have challenging long range and short range characteristics. The Coulomb, inter-molecular and nuclear interactions all share this challenge. We review one successful approach, the ab-initio no-core shell model (NCSM), and survey its applications. Recent results with our method appear in a companion paper in these proceedings [1].

For further motivation, we present in Fig. 1 a sketch of the overlaps between ab-initio methods, such as the NCSM, and some other areas of current intensive research. Our own efforts have touched on a variety of the interfaces sketched in Fig. 1.

\section{NO-CORE SHELL-MODEL}

The NCSM [2-7] is based on an effective Hamiltonian derived from realistic "bare" interactions and acting within a finite Hilbert space. All $A$-nucleons are treated on an equal

\footnotetext{
*Supported in part by USDOE grant DE-FG-02 87ER40371

${ }^{\dagger}$ Supported in part by NSF grant PHY0070858

${ }^{\ddagger}$ This work was partly performed under the auspices of the U. S. Department of Energy by the University of California, Lawrence Livermore National Laboratory under contract No. W-7405-Eng-48

${ }^{\S}$ Supported in part by DOE grants DE-FC02-01ER41187 and DE-FG03-00ER41132
} 
footing. The approach is both computationally tractable and demonstrably convergent to the exact result of the full (infinite) Hilbert space.

Initial investigations used two-body interactions [2] based on a G-matrix approach. Later, we implemented the Lee-Suzuki procedure [8] to derive two-body and three-body effective interactions based on realistic NN and NNN interactions.

\subsection{Effective Hamiltonian}

For pedagogical purposes, we outline the NCSM approach with NN interactions alone and point the reader to the literature for the extensions to include NNN interactions. We begin with the purely intrinsic Hamiltonian for the $A$-nucleon system, i.e.,

$H_{A}=T_{\mathrm{rel}}+\mathcal{V}=\frac{1}{A} \sum_{i<j}^{A} \frac{\left(\vec{p}_{i}-\vec{p}_{j}\right)^{2}}{2 m}+\sum_{i<j=1}^{A} V_{\mathrm{N}}\left(\vec{r}_{i}-\vec{r}_{j}\right)$

where $m$ is the nucleon mass and $V_{\mathrm{N}}\left(\vec{r}_{i}-\vec{r}_{j}\right)$, the NN interaction, with both strong and electromagnetic components. Note the absence of a phenomenological single-particle potential. We may use either coordinate-space NN potentials, such as the Argonne potentials [9] or momentum-space dependent NN potentials, such as the CD-Bonn [10].

Next, we add the center-of-mass HO Hamiltonian to the Hamiltonian (1) $H_{\mathrm{CM}}=T_{\mathrm{CM}}+$ $U_{\mathrm{CM}}$, where $U_{\mathrm{CM}}=\frac{1}{2} A m \Omega^{2} \vec{R}^{2}, \vec{R}=\frac{1}{A} \sum_{i=1}^{A} \vec{r}_{i}$. In the full Hilbert space the added $H_{\mathrm{CM}}$ term has no influence on the intrinsic properties. However, when we introduce our cluster approximation below, the added $H_{\mathrm{CM}}$ term facilitates convergence to exact results with increasing basis size. The modified Hamiltonian, with a pseudo-dependence on the HO frequency $\Omega$, can be cast into the form

$H_{A}^{\Omega}=H_{A}+H_{\mathrm{CM}}=\sum_{i=1}^{A}\left[\frac{\vec{p}_{i}^{2}}{2 m}+\frac{1}{2} m \Omega^{2} \vec{r}_{i}^{2}\right]+\sum_{i<j=1}^{A}\left[V_{\mathrm{N}}\left(\vec{r}_{i}-\vec{r}_{j}\right)-\frac{m \Omega^{2}}{2 A}\left(\vec{r}_{i}-\vec{r}_{j}\right)^{2}\right]$.

In the spirit of Da Providencia and Shakin [12] and Lee, Suzuki and Okamoto [8,11], we introduce a unitary transformation, which is able to accommodate the short-range two-body correlations in a nucleus, by choosing an antihermitian operator $S$, acting only on intrinsic coordinates, such that

$\mathcal{H}=e^{-S} H_{A}^{\Omega} e^{S}$

In our approach, $S$ is determined by the requirements that $\mathcal{H}$ and $H_{A}^{\Omega}$ have the same symmetries and eigenspectra over the subspace $\mathcal{K}$ of the full Hilbert space. In general, both $\mathrm{S}$ and the transformed Hamiltonian are $A$-body operators. Our simplest, non-trivial approximation to $\mathcal{H}$ is to develop a two-body $(a=2)$ effective Hamiltonian, where the upper bound of the summations " $A$ " is replaced by " $a$ ", but the coefficients remain unchanged. The next improvement is to develop a three-body effective Hamiltonian, $(a=3)$. This approach consists then of an approximation to a particular level of clustering with $a \leq A$.

$\mathcal{H}=\mathcal{H}^{(1)}+\mathcal{H}^{(a)}=\sum_{i=1}^{A} h_{i}+\frac{\left(\begin{array}{l}A \\ 2\end{array}\right)}{\left(\begin{array}{c}A \\ a\end{array}\right)\left(\begin{array}{l}a \\ 2\end{array}\right)} \sum_{i_{1}<i_{2}<\ldots<i_{a}}^{A} \tilde{V}_{i_{1} i_{2} \ldots i_{a}}$ 
with

$\tilde{V}_{12 \ldots a}=e^{-S^{(a)}} H_{a}^{\Omega} e^{S^{(a)}}-\sum_{i=1}^{a} h_{i}$

and $S^{(a)}$ is an $a$-body operator; $H_{a}^{\Omega}=h_{1}+h_{2}+h_{3}+\ldots+h_{a}+V_{a}$, and $V_{a}=\sum_{i<j}^{a} V_{i j}$. Note that there is no sum over " $a$ " in Eq. (4). Also, we adopt the HO basis states that are eigenstates of the one-body Hamiltonian $\sum_{i=1}^{A} h_{i}$.

If the full Hilbert space is divided into a finite model space ("P-space") and a complementary infinite space ("Q-space"), using the projectors $P$ and $Q$ with $P+Q=1$, it is possible to determine the transformation operator $S_{a}$ from the decoupling condition

$Q_{a} e^{-S^{(a)}} H_{a}^{\Omega} e^{S^{(a)}} P_{a}=0$

and the simultaneous restrictions $P_{a} S^{(a)} P_{a}=Q_{a} S^{(a)} Q_{a}=0$. Note that $a$-nucleon-state projectors $\left(P_{a}, Q_{a}\right)$ appear in Eq. (6). Their definitions follow from the definitions of the $A$-nucleon projectors $P, Q$. The unitary transformation and decoupling condition, introduced by Suzuki and Okamoto and referred to as the unitary-model-operator approach (UMOA) [13], has a solution that can be expressed in the following form

$S^{(a)}=\operatorname{arctanh}\left(\omega-\omega^{\dagger}\right)$

with the operator $\omega$ satisfying $\omega=Q_{a} \omega P_{a}$, and solving its own decoupling equation,

$Q_{a} e^{-\omega} H_{a}^{\Omega} e^{\omega} P_{a}=0$

Let us also note that $\bar{H}_{\mathrm{a}-\mathrm{eff}}=P_{a} e^{-S^{(a)}} H_{a}^{\Omega} e^{S^{(a)}} P_{a}$ leads to the relation

$\bar{H}_{\mathrm{a}-\mathrm{eff}}=\left(P_{a}+\omega^{\dagger} \omega\right)^{-1 / 2}\left(P_{a}+P_{a} \omega^{\dagger} Q_{a}\right) H_{a}^{\Omega}\left(Q_{a} \omega P_{a}+P_{a}\right)\left(P_{a}+\omega^{\dagger} \omega\right)^{-1 / 2}$.

Given the eigensolutions, $H_{a}^{\Omega}|k\rangle=E_{k}|k\rangle$, then the operator $\omega$ can be determined from

$\left\langle\alpha_{Q}|\omega| \alpha_{P}\right\rangle=\sum_{k \in \mathcal{K}}\left\langle\alpha_{Q} \mid k\right\rangle\left\langle\tilde{k} \mid \alpha_{P}\right\rangle$

where we denote by tilde the inverted matrix of $\left\langle\alpha_{P} \mid k\right\rangle$, i.e., $\sum_{\alpha_{P}}\left\langle\tilde{k} \mid \alpha_{P}\right\rangle\left\langle\alpha_{P} \mid k^{\prime}\right\rangle=\delta_{k, k^{\prime}}$ and $\sum_{k}\left\langle\alpha_{P}^{\prime} \mid \tilde{k}\right\rangle\left\langle k \mid \alpha_{P}\right\rangle=\delta_{\alpha_{P}^{\prime}, \alpha_{P}}$, for $k, k^{\prime} \in \mathcal{K}$. In the relation (10), $\left|\alpha_{P}\right\rangle$ and $\left|\alpha_{Q}\right\rangle$ are the model-space and the Q-space basis states, respectively, and $\mathcal{K}$ denotes a set of $d_{P}$ eigenstates, whose properties are reproduced in the model space, with $d_{P}$ equal to the dimension of the model space.

With the help of the solution for $\omega(10)$ we obtain a simple expression for the matrix elements of the hermitian effective Hamiltonian

$$
\begin{aligned}
\left\langle\alpha_{P}\left|\bar{H}_{\mathrm{a}-\mathrm{eff}}\right| \alpha_{P}^{\prime}\right\rangle= & \sum_{k \in \mathcal{K}} \sum_{\alpha_{P}^{\prime \prime}} \sum_{\alpha_{P}^{\prime \prime \prime}}\left\langle\alpha_{P}\left|\left(P_{a}+\omega^{\dagger} \omega\right)^{-1 / 2}\right| \alpha_{P}^{\prime \prime}\right\rangle\left\langle\alpha_{P}^{\prime \prime} \mid \tilde{k}\right\rangle E_{k}\left\langle\tilde{k} \mid \alpha_{P}^{\prime \prime \prime}\right\rangle \\
& \times\left\langle\alpha_{P}^{\prime \prime \prime}\left|\left(P_{a}+\omega^{\dagger} \omega\right)^{-1 / 2}\right| \alpha_{P}^{\prime}\right\rangle .
\end{aligned}
$$

For computation of the matrix elements of $\left(P_{a}+\omega^{\dagger} \omega\right)^{-1 / 2}$, we can use the relation

$$
\left\langle\alpha_{P}\left|\left(P_{a}+\omega^{\dagger} \omega\right)\right| \alpha_{P}^{\prime \prime}\right\rangle=\sum_{k \in \mathcal{K}}\left\langle\alpha_{P} \mid \tilde{k}\right\rangle\left\langle\tilde{k} \mid \alpha_{P}^{\prime \prime}\right\rangle
$$


We note that in the limit $a \rightarrow A$, we obtain the exact solutions for $d_{P}$ states of the full problem for any finite basis space, with flexibility for choice of physical states subject to certain conditions [14].

On account of our cluster approximation a dependence of our results on $N_{m}$ and on $\Omega$ arises. For a fixed cluster size, the smaller the basis space, the larger the dependence on $\Omega$. The residual $N_{m}$ and $\Omega$ dependences can be used to infer the uncertainty in our results.

The model space $P_{2}$ is defined by the maximal number of allowed $\mathrm{HO}$ quanta of the $A$-nucleon basis states $N_{\mathrm{m}}$ from the condition $2 n+l \leq N_{\mathrm{m}}-N_{\text {spsmin }}$, where $N_{\text {spsmin }}$ denotes the minimal possible $\mathrm{HO}$ quanta of the spectators, i.e., nucleons not affected by the interaction process. For example, ${ }^{10} \mathrm{~B}, N_{\text {spsmin }}=4$ as there are 6 nucleons in the $0 p$-shell in the lowest $\mathrm{HO}$ configuration and, e.g., $N_{\mathrm{m}}=N_{\text {spsmin }}+2+N_{\max }$, where $N_{\max }$ represents the maximum $\mathrm{HO}$ quanta of the many-body excitation above the unperturbed ground-state configuration. For ${ }^{10} \mathrm{~B}, N_{\mathrm{m}}=12$ for an $N_{\max }=6$ or " $6 \hbar \Omega$ " calculation.

In order to construct the operator $\omega(10)$ we need to select the set of eigenvectors $\mathcal{K}$. We select the lowest states obtained in each two-body channel. It turns out that these states also have the largest overlap with the model space for the range of $\hbar \Omega$ we have investigated and the P-spaces we select. Their number is given by the number of basis states satisfying $2 n+l \leq N_{\mathrm{m}}-N_{\text {spsmin }}$.

We input the effective Hamiltonian, now consisting of a relative 2-body operator and the pure $H_{C M}$ term introduced earlier, into an m-scheme Lanczos diagonalization process to obtain the P-space eigenvalues and eigenvectors. At this stage we also add the term $H_{C M}$ again with a large positive coefficient to separate the physically interesting states with $0 S \mathrm{CM}$ motion from those with excited CM motion. We retain only the states with pure $0 S \mathrm{CM}$ motion when evaluating observables. All observables that are expressible as functions of relative coordinates, such as the rms radius and radial densities, are then evaluated free of CM motion effects.

We close our presentation on the theoretical framework with the observation that all observables require the same transformation as implemented on the Hamiltonian. To date, we have found rather small effects on the rms radius operator when we transformed it to a P-space effective rms operator at the $\mathrm{a}=2$ cluster level [6]. On the other hand, substantial renormalization was observed for the kinetic energy operator when using the $\mathrm{a}=2$ transformation to evaluate its expectation value [15].

\section{ILLUSTRATIVE RESULTS AND DISCUSSION}

Here we will present only results at the $a=2$ cluster level. Fig. 2 depicts how the binding energy for ${ }^{3} \mathrm{H}$ converges to the exact result for different choices of the basis space size (governed by $N_{\max }$ ) and the single particle wave function length scale (governed by $\hbar \Omega$ ) [6]. If we select $\hbar \Omega$ with the criteria of least sensitivity to $N_{\max }$, we achieve excellent results at rather low values of $N_{\max }$.

We present the $6 \hbar \omega$ results for ${ }^{12} \mathrm{C}$ in Fig. 3. These results extend those presented through $4 \hbar \Omega$ in Ref. [6]. We note the reasonable convergence of the states dominated by $0 \hbar \Omega$ configurations while the low-lying experimental "alpha-cluster" states, or "intruder states" in our language, will require significantly larger model spaces to converge. 


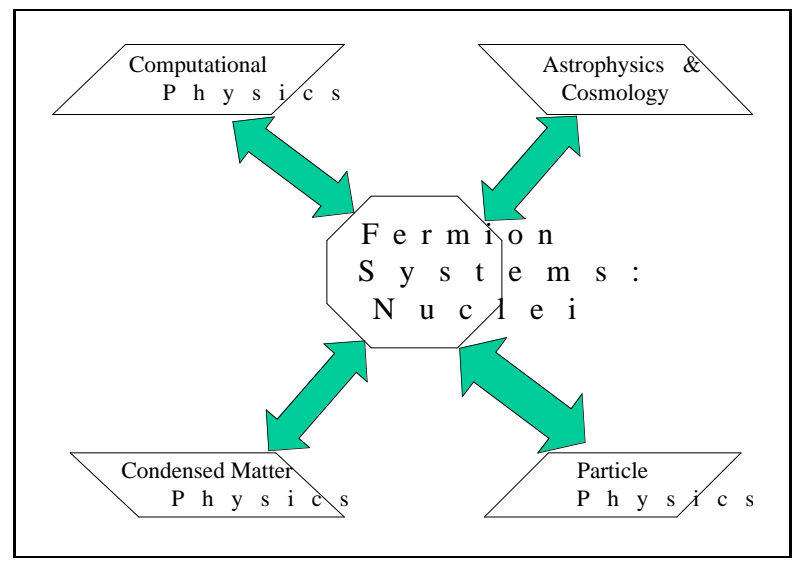

Figure 1. Illustration of overlaps between $a b$ initio nuclear many-body theory with other current research areas.

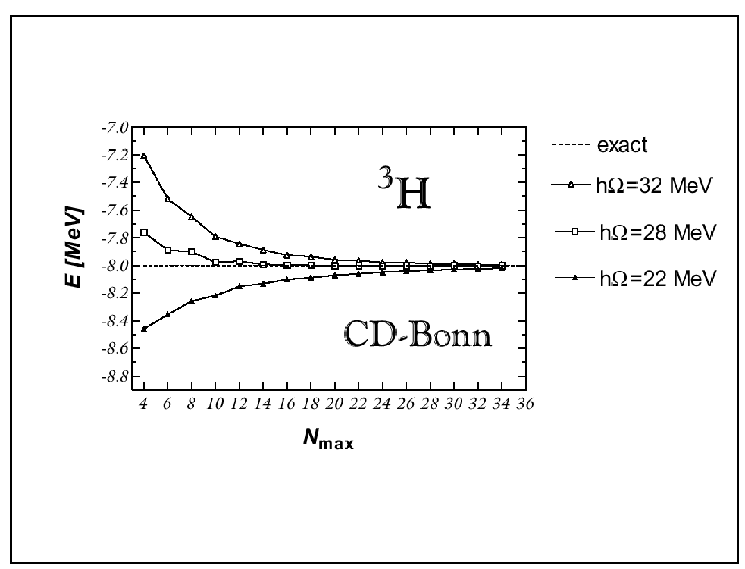

Figure 2. The dependence of the ${ }^{3} \mathrm{H}$ ground-state energy on number of $\mathrm{HO}$ quanta in the basis space for the CD-Bonn interaction at three values of $\hbar \Omega$.

In Fig. 4 we present, for the first time, initial results for ${ }^{16} \mathrm{O}$. Since the binding energy showed a strong dependence on $N_{\max }$, while the binding of the first excited $0^{+}$state exhibits far less sensitivity, we portray our spectra beginning with the first excited $0^{+}$ state and shift theory to agree with that experimental state. All known excited states are observed in the NCSM. To gauge convergence, we portray the RMS change in the depicted eigenvalues when progressing from the $2 \hbar \Omega$ through the $6 \hbar \Omega$ basis spaces. It is encouraging to note that the RMS change in eigenvalues decreases by a factor of two over the range of results presented. We conclude that the residual disagreements with experiment, as indicated by RMS deviation of more than $1 \mathrm{MeV}$ between the $6 \hbar \Omega$ results and experiment, are unlikely to be overcome in larger model spaces. Instead, we conclude that we need NNN interactions to explain most of the remaining differences.

In view of the great interest in nuclei far from stability, we evaluate the spectra of ${ }^{16} \mathrm{C}$ using the same effective Hamiltonian as we employed for ${ }^{16} \mathrm{O}$. Our initial spectra above the $0^{+}$ground state for ${ }^{16} \mathrm{C}$ through the $4 \hbar \Omega$ basis space consists of the states $2^{+}, 0^{+}, 2^{+}, 3^{+}$and $4^{+}$at excitation energies of $2.06,3.25,4.66,4.77$ and $5.66 \mathrm{MeV}$ respectively. These results are in reasonable agreement with the experimental excited state spectra of $2^{+},\left(0^{+}\right), 2,3^{(+)}$and $4^{+}$at excitation energies of $1.77,3.03,3.99,4.09$ and 4.14 $\mathrm{MeV}$ respectively. It will be interesting to evaluate the $\mathrm{B}(\mathrm{E} 2)$ for the transition from the first excited state to compare with the reported anomalous hindering of this transtion $[16]$.

In an initial application to multiquark systems [17], we have fit a QCD-inspired phenomenological non-relativistic effective Hamitonian to the low-lying Charmonia spectra in a $10 \hbar \Omega$ model space. We adjusted the charm quark mass, coupling constants and $\hbar \Omega$ in two separate fits to the lowest four negative orbital parity and lowest four positive orbital parity states. The fits are competitive with standard constituent quark models.

We then solve for the masses of the tetraquark states in the same model space, and 


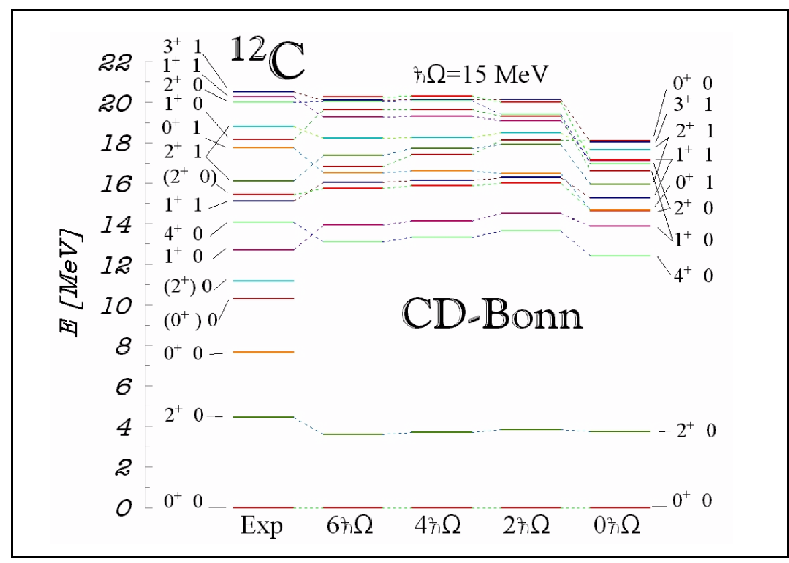

Figure 3. Low-lying spectra of ${ }^{12} \mathrm{C}$ from the CD-Bonn interaction at the $\mathrm{a}=2$ cluster approximation in the NCSM. The spectra are shifted to align the calculated ground state with the exerimental ground state.

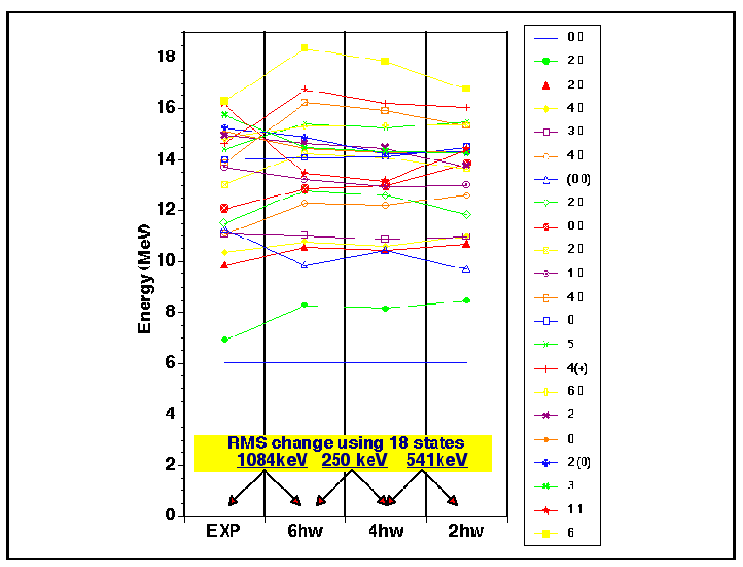

Figure 4. Low-lying ${ }^{16} \mathrm{O}$ states from the CD-Bonn interaction at the $\mathrm{a}=2$ cluster approximation in the NCSM with $\hbar \Omega=$ $15 \mathrm{MeV}$. The spectra are aligned with the experimental first excited $0^{+}$state.

we obtain states whose quantum numbers indicate they are bound with respect to the closest allowed threshold for decay by breakup. Net binding in the range of 50-200 $\mathrm{MeV}$ are indicated by these results. Note that we use projection techniques to assure that the tetraquark states are global color singlets. Apparently, few previous experiments had the sensitivity to observe these exotic systems so it will be interesting to see if future experiments observe these "all-charm tetraquarks."

To close, we mention a few of our efforts to address unsolved problems, some of which sit squarely on the interfaces depicted in Fig. 1. In the near term, we are addressing comparisons of spherical Hartree-Fock with NCSM results [18], extrapolation methods to extend the NCSM to larger spaces [19], NCSM applications to the halo nucleus ${ }^{6} \mathrm{He}$ comparing with neutron-neutron correlation data from breakup reactions [20], extending NCSM to evaluate effective operators for additional observables [21], NCSM applications to $A=48$ nuclei with intentions of addressing nuclear double beta-decay [22], NCSM applications to $A=56$ nuclei addressing statistical properties relevant for nuclear astrophysics [23], applications to quantum field theory [24] and to multiquark systems with relativistic Hamiltonians more closely aligned with QCD [25].

Clearly, the road ahead is promising and we anticipate many additional forefront applications of the NCSM in the future.

\section{REFERENCES}

1. B.R. Barrett, P. Navratil, A. Nogga, W.E. Ormand, and J.P. Vary, see contribution in these proceedings.

2. D. C. Zheng, B. R. Barrett, L. Jaqua, J. P. Vary, and R. L. McCarthy, Phys. Rev. C 48, 1083 (1993); D. C. Zheng, J. P. Vary, and B. R. Barrett, Phys. Rev. C 50, 2841 
(1994); D. C. Zheng, B. R. Barrett, J. P. Vary, W. C. Haxton, and C. L. Song, Phys. Rev. C 52, 2488 (1995).

3. P. Navrátil and B. R. Barrett, Phys. Rev. C 54, 2986 (1996); Phys. Rev. C 57, 3119 (1998).

4. P. Navrátil and B. R. Barrett, Phys. Rev. C 57, 562 (1998).

5. P. Navrátil and B. R. Barrett, Phys. Rev. C 59, 1906 (1999); P. Navrátil, G. P. Kamuntavičius and B. R. Barrett, Phys. Rev. C 61, 044001 (2000).

6. P. Navrátil, J. P. Vary and B. R. Barrett, Phys. Rev. Lett. 84, 5728 (2000); Phys. Rev. C 62, 054311 (2000).

7. P. Navrátil, J. P. Vary, W. E. Ormand and B. R. Barrett, Phys. Rev. Lett. 87, 172502 (2001). E. Caurier, P. Navrátil, W. E. Ormand and J. P. Vary, Phys. Rev. C 64, 051301 (2001).

8. K. Suzuki and S.Y. Lee, Prog. Theor. Phys. 64, 2091 (1980); K. Suzuki, Prog. Theor. Phys. 68, 246 (1982).

9. R. B. Wiringa, V. G. J. Stoks and R. Schiavilla, Phys. Rev. C 51, 38 (1995).

10. R. Machleidt, F. Sammarruca and Y. Song, Phys. Rev. C 53, 1483 (1996); R. Machleidt, Phys. Rev. C 63, 024001 (2001).

11. K. Suzuki, Prog. Theor. Phys. 68, 246 (1982); K. Suzuki and R. Okamoto, Prog. Theor. Phys. 70, 439 (1983).

12. J. Da Providencia and C. M. Shakin, Ann. of Phys. 30, 95 (1964).

13. K. Suzuki, Prog. Theor. Phys. 68, 1999 (1982); K. Suzuki and R. Okamoto, Prog. Theor. Phys. 92, 1045 (1994).

14. C.P. Viazminsky and J.P. Vary, J. Math. Phys., 42, 2055(2001).

15. H. Kamada, et. al, Phys. Rev. C 64 044001(2001).

16. N. Imai, et. al, Riken preprint AF-NP-452, August 2003.

17. R.J. Lloyd and J.P. Vary, "All-Charm Tetraquarks," hep-ph/0311179.

18. M.A. Hasan, J.P. Vary, and P. Navrátil, Phys. Rev. C to appear.

19. H. Zhan, A. Nogga, B.R. Barrett, J.P. Vary and P. Navrátil, Phys. Rev. C to appear. 20. O. Atramentov, J.P. Vary and P. Navrátil, in preparation.

21. I. Stetcu, et. al, in preparation.

22. S. Popescu, S. Stoica and J.P. Vary, in preparation.

23. B. Shehadeh, M.A. Hasan and J.P. Vary, in preparation.

24. D. Chakrabarti, A. Harindranath and J.P. Vary, hep-ph/0309317, Phys. Rev. D. to appear; D. Chakrabarti, A. Harindranath, L. Martinovic, G. Pivovarov and J.P. Vary, hep-th/0310290; D. Chakrabarti, A. Harindranath, L. Martinovic and J.P. Vary, hepth/0309263.

25. R.J. Lloyd, J.R. Spence and J.P. Vary, in preparation. 